Supporting Information

\title{
Mode Coupling of Whispering Gallery Modes through Organic Semiconductor
}

\section{Thin Films}

Akihiro Kameda, ${ }^{\dagger}$ Sunao Shimomoto,${ }^{\dagger}$ Hiroyuki Tajima, ${ }^{\dagger}$ Jun-ichi Yamada,${ }^{\dagger}$ Tokuji Yokomatsu, ${ }^{\dagger}$

Kazusuke Maenaka, ${ }^{\dagger}$ Takeshi Komino*†

${ }^{\dagger}$ Graduate School of Science, University of Hyogo, Ako-gun, Hyogo 678-1297, Japan

${ }^{\ddagger}$ Graduate School of Engineering, University of Hyogo, Himeji, Hyogo 671-2201, Japan

*E-mail: komino@sci.u-hyogo.ac.jp 
The observation field area in spectrum acquisitions. We created a laser spot (Nd:YAG, $355 \mathrm{~nm})$ on a 230-nm-thick BSB-Cz thin film fabricated on a non-fluorescent glass substrate, which was mounted on a microscope stage. Figure S1a shows the schematic of the setup. Positioning of the laser spot was controlled by horizontal and vertical movement of a quartz lens.

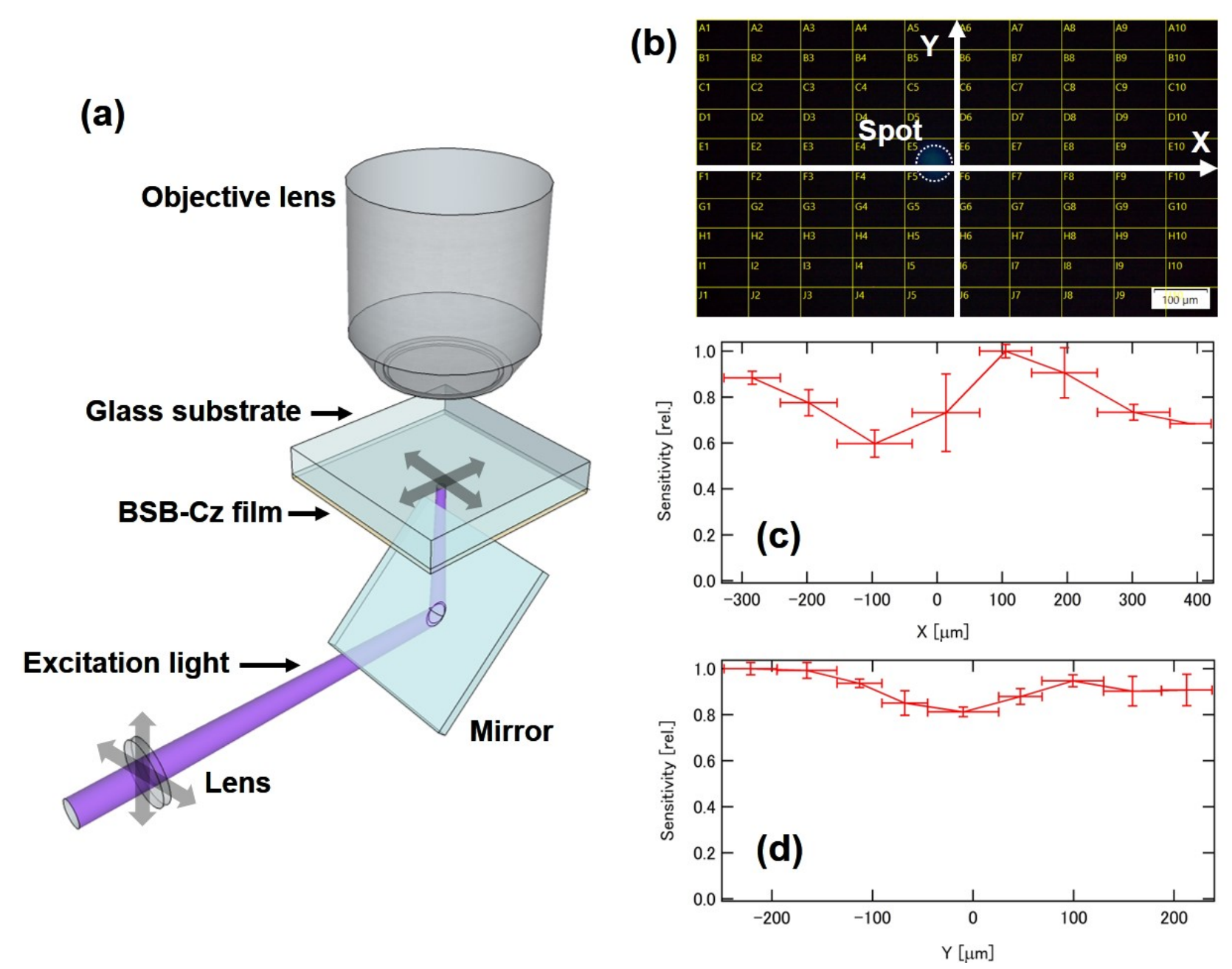

Figure S1. (a) Experimental setup for the sensitivity estimation. (b) A PL spot on a BSB-Cz thin film in the field of view of the microscope. The image was obtained by using a software (Cell Sense, Olympus Co.). (c) The distribution of sensitivity along the $\mathrm{X}$ axis $(\mathrm{Y}=0)$, and (d) the distribution of sensitivity along the $\mathrm{Y}$ axis $(\mathrm{X}=0)$. These axes correspond to the white lines exhibited in $(\mathrm{b})$.

With dark field images (Fig. S1b), we acquired a maximum photon number in the PL spot and the XY coordinates by using software package (Cell Sense, Olympus Co.). Considering the linear relationship between a photon number and a PL intensity, the maximum photon number $\left(N_{\mathrm{p}}\right)$ is proportional to the maximum PL intensity in the field of view of the microscope. Maintaining the 
geometry, we switched the optical path in the microscope to the spectrum acquisition mode and measured spectral intensity $\left(I_{\mathrm{S}}\right)$ to estimate a sensitivity in spectra measurements. The sensitivity was calculated from the ratio $I_{\mathrm{s}} / N_{\mathrm{p}}$. Figure $\mathrm{S} 1 \mathrm{c}$ shows the sensitivities along $\mathrm{X}$ axis $(\mathrm{Y}=0$ [center of the horizontal axis]), while Fig. S1d shows those along $\mathrm{Y}$ axis $(\mathrm{X}=0$ [center of the vertical axis]). Vertical axes in Figs. S1c and d were normalized at the maximum sensitivities. As seen in Figs. S1c and d, the sensitivities are uniformly distributed. Considering the large excitation laser spot of $400 \mu \mathrm{m}$ in the PL spectra measurements described in the main text, the acquired spectra reflect the PL emission from the total area of the field of view.

\section{Photoluminescence spectra of a BSB-Cz film fabricated on a substrate without a microdisk}

structure. Figure S2 shows the photoluminescence (PL) spectra of BSB-Cz fabricated on a substrate without a microdisk structure. The film thickness of BSB-Cz was $230 \mathrm{~nm}$. Spectral narrowing was observed at $477 \mathrm{~nm}$. We attribute this peak to amplified spontaneous emission (ASE). This result suggests that a peak at $477 \mathrm{~nm}$ with full width at half maximum (FWHM) of over $10 \mathrm{~nm}$ did not originate from whispering gallery mode (WGM) even in samples with microdisk structures.

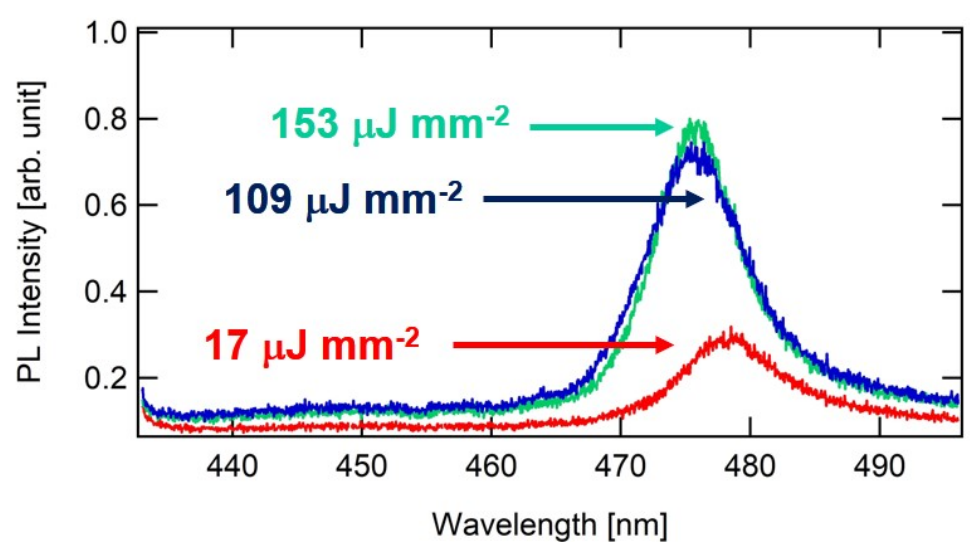

Figure S2. PL spectra obtained in a BSB-Cz thin film (without microdisks). The excitation intensities are displayed in the figure; the intensities are the same as those in the samples with microdisks shown in the main text. 
PL spectra of BSB-Cz films with the microdisk diameter of $4 \boldsymbol{\mu m}$. Figure S3 shows the PL spectra of BSB-Cz films fabricated on a substrate having microdisks with the diameter of $4 \pm 1 \mathrm{~mm}$. The film thickness was $230 \mathrm{~nm}$. $L$ is a distance between two adjacent microdisks. While a small sharp peak appeared in $L=2 \mu \mathrm{m}$ (Fig. S2a), a significant periodic structure was not observed in these samples. We tested other samples having some different thickness ranging from 70 to $115 \mathrm{~nm}$, but the results were similar to Fig. S3.
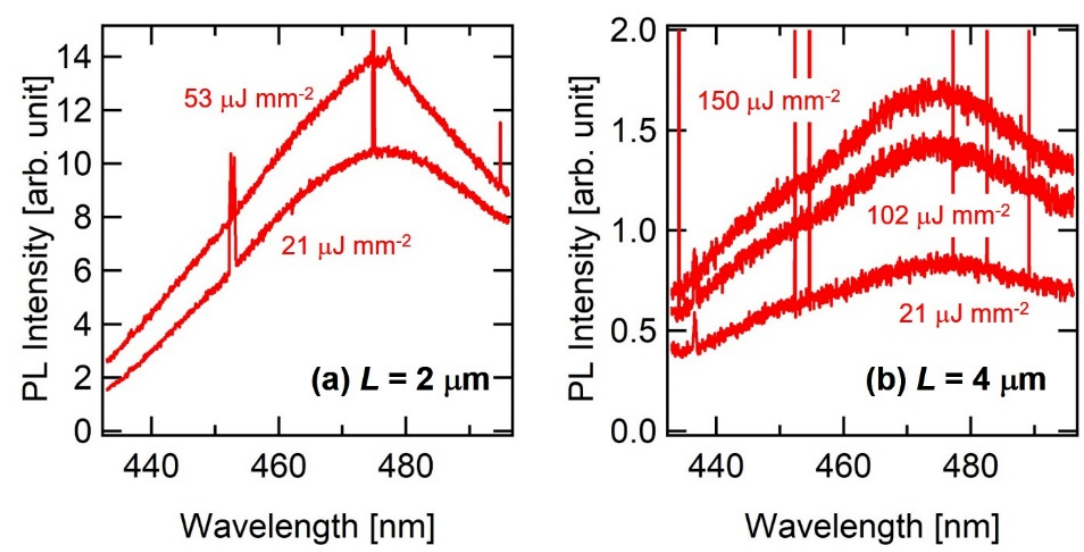

Figure S3. PL spectra obtained in a BSB-Cz thin film on substrates having microdisks of $4 \mu \mathrm{m}$ diameter with (a) $L=2 \mu \mathrm{m}$, and (b) $L=4 \mu \mathrm{m}$. In the result of $L=2 \mu \mathrm{m}$, photodegradation occurred when the samples were excited with high intensity above approximately $100 \mu \mathrm{J} \mathrm{mm}^{-2}$.

The optical simulation for a 115-nm-thick BSB-Cz microdisk. Figure S4 shows the simulated spectra of electric field. The condition for the simulation was the same as the main text but the film thickness $(115 \mathrm{~nm})$. TE mode intensity decreased with decreasing a film thickness $(t)$ of BSB-Cz (cf. Fig. 4), while TM mode intensity was enhanced by decreasing $t$. The trend for the dependence of electric field on $t$ is similar to the dependence of peak intensity for WGM on $t$, so we conclude that TE mode is dominant in the experimentally obtained the WGM spectra. 

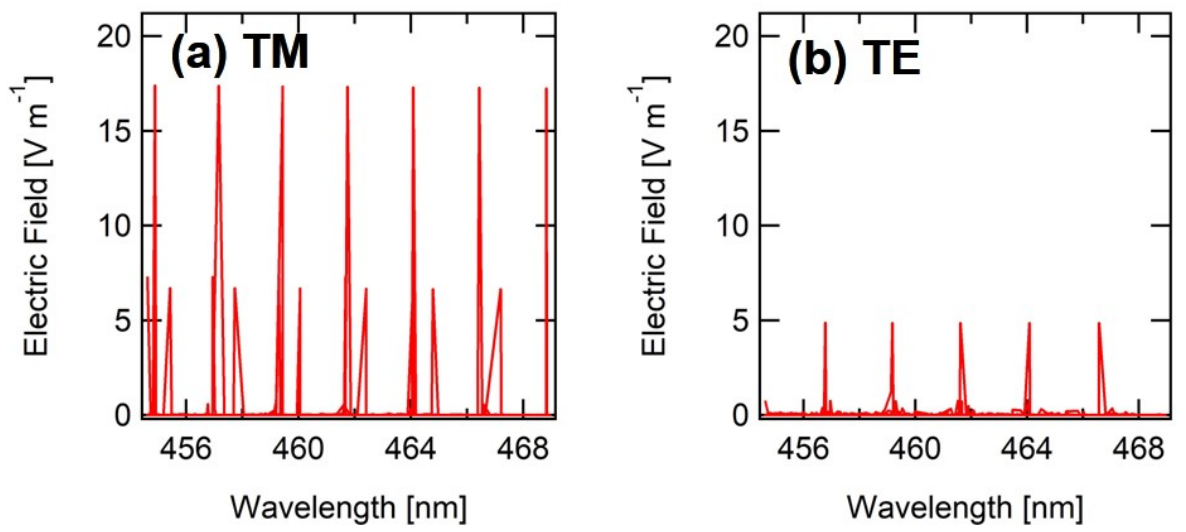

Figure S4. Simulation results for WGM spectra in (a) TM mode and (b) TE mode.

PL spectra of 115-nm-thick BSB-Cz films having the microdisk diameter of $15 \mu \mathrm{m}$. Figure S5 shows the PL spectra of 115-nm-thick BSB-Cz thin films, which were fabricated on substrates having microdisks with the diameter of $15 \mu \mathrm{m}$.
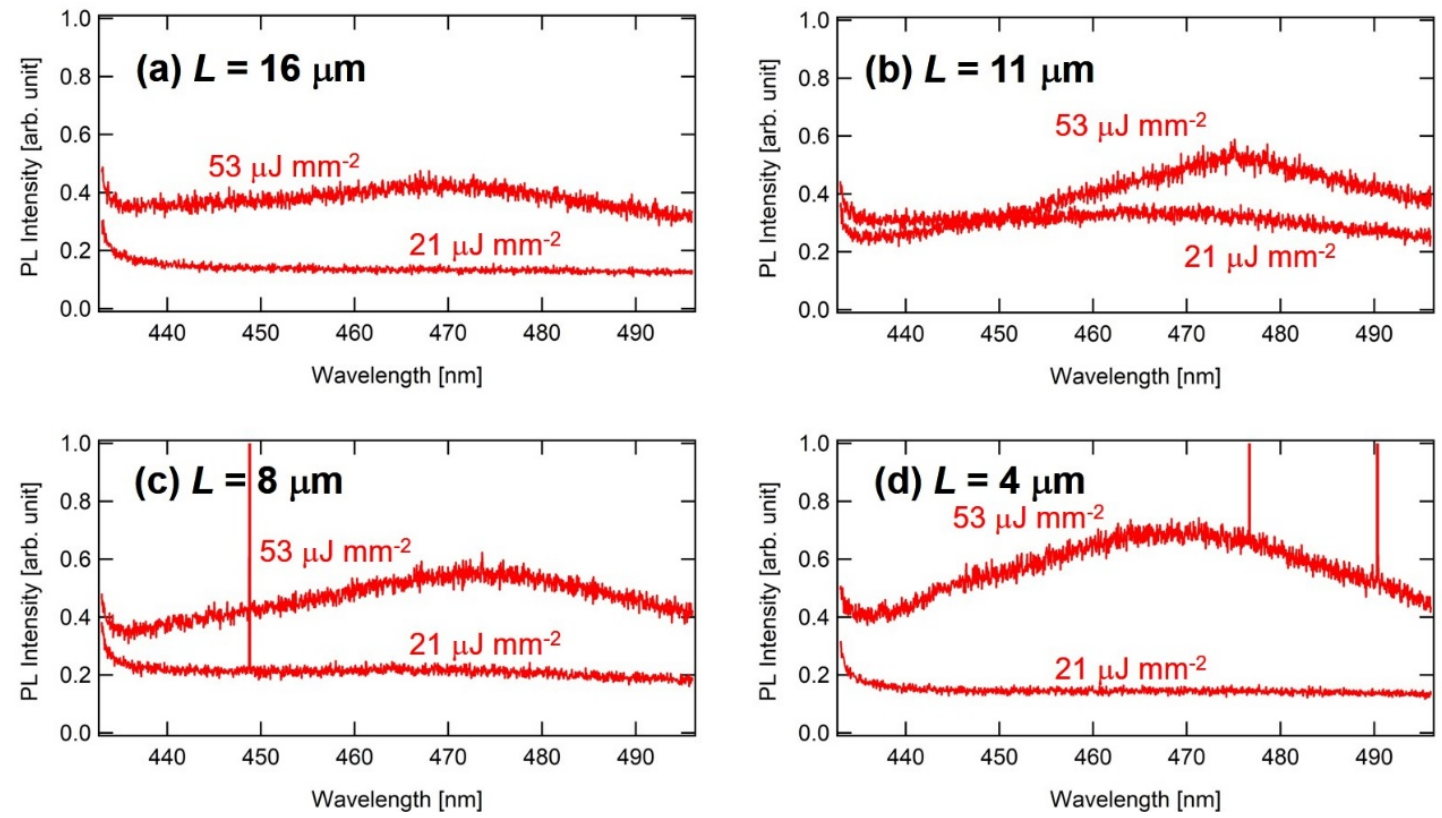

Figure S5. PL spectra of the 115-nm-thick BSB-Cz microdisks having $L$ of (a) $16 \mu \mathrm{m}$ (b) $11 \mu \mathrm{m}$ (c) 8 $\mu \mathrm{m}$, and (d) $4 \mu \mathrm{m}$. Excitation intensities were indicated in each spectrum. No WGM resonance was observed in each spectrum. Spike peaks and high intensity at $433 \mathrm{~nm}$ are due to cosmic ray and stray radiation, respectively. At high intensity excitation above approximately $100 \mu \mathrm{J} \mathrm{mm}^{-2}$, the samples were photodegraded. This trend is similar to a BSB-Cz microdisks with the diameter of $4 \mu \mathrm{m}$ (Fig. S3). We ascribe the photodegradation to heat accumulation when efficient PL emission does not occur.

Estimation of penetration depth for an evanescent wave around a microdisk. At a flat interface S-5 
between two planer media, the penetration depth of an evanescent wave $(\Delta p)$ is calculated by $\Delta p=$ $\frac{\lambda}{2 \pi n_{1}}\left(\cos ^{2} \theta-\left(\frac{n_{1}}{n_{2}}\right)^{2}\right)^{-\frac{1}{2}}$, where $\lambda$ is wavelength, $\theta$ is angle of incidence (between interface and incident light), $n_{1}$ and $n_{2}$ are refractive indices of media for the incidence side and the transmission side, respectively. If we assume that a round-shaped interface of an outside edge in WGM resonators is approximately flat, the above equation estimates $\Delta p$ of $44 \mathrm{~nm}$ for $\lambda=480 \mathrm{~nm}$. Herein, we assumed that $\theta$ is less than $1^{\circ}$, because an angle between a circle having a diameter of $10 \mu \mathrm{m}$ and the tangential line is less than $1^{\circ}$ when we limit the area in the vicinity of crossing point within $480 \mathrm{~nm}$; we consider the circle a regular polygon with a side $480 \mathrm{~nm}$ long.
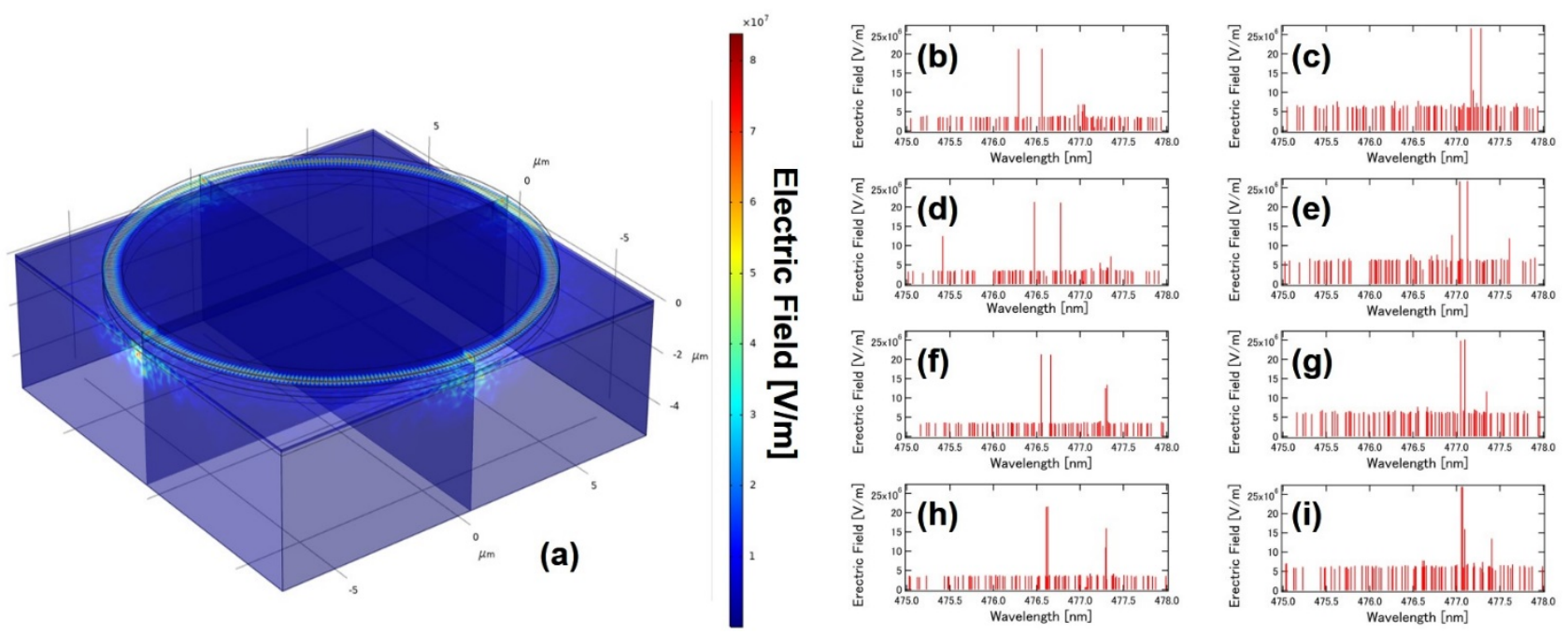

Figure S6. (a) The electric field distribution image of the transverse magnetic (TE) mode at 476.23 $\mathrm{nm}$ in $L=25 \mathrm{~nm}$. The simulated electric field spectra of $L=25 \mathrm{~nm}$ (b and c), $50 \mathrm{~nm}$ (d and e), $100 \mathrm{~nm}$ ( $f$ and $g$ ), and $200 \mathrm{~nm}$ (h and i), respectively. The figures of b, d, f, and h are for the TE modes, while c, e, $g, i$ are for the TM modes.

To test whether or not a peak splitting occurs by overlapping of evanescent waves, an optical simulation for three-dimensional structures was conducted using a commercial software package (Multiphysics ver. 5.4 and Wave Optics Module ver. 5.4, Comsol Co.). The model geometry is shown in Fig. S6a. A 230-nm-thick organic microdisk covers over the top surface of a 570-nm-thick $\mathrm{SiO}_{2}$ microdisk. The diameter of the microdisks was set to $15 \mu \mathrm{m}$. The stacked microdisks were mounted on a glass substrate. We applied the periodic boundary condition to the $\mathrm{x}$ and $\mathrm{y}$ boundaries in the 
structure. The refractive indices of the $\mathrm{SiO}_{2}$ (and glass), the organic material and air were set at 1.5, 2.0 and 1.0 , respectively. To reduce the computational cost, we put a perfectly matched layer in the center of the organic microdisk with the diameter of $13.72 \mu \mathrm{m}$, which is the condition to adequately generate the WGM with $m=170$. The maximum size of the elements in the WGM path was set to be $0.1 \mu \mathrm{m}$. Using the geometry, we sought an eigenmode frequency. Figure S6 shows the simulation results. The spectra exhibited small peaks $\left(<7 \times 10^{6} \mathrm{~V} \mathrm{~m}^{-1}\right)$ and larger peaks. The larger peaks correspond to WGM, while the small peaks originate from the modes other than WGM. The WGM peaks exhibited the peak splitting especially in the region of $L<100 \mathrm{~nm}$, and the magnitude of peak splitting becomes larger with decreasing $L$. This result is consistent with the above estimation of the penetration depth (approximately $44 \mathrm{~nm}$ ).

\section{Cross-sectional profiles of $\mathrm{SiO}_{2}$ microdisks and surface roughness of $\mathrm{SiO}_{2}$ and $\mathrm{BSB}-\mathrm{Cz}$ layers. $\mathrm{A}$} three-dimensional structure of the microdisks was investigated by a coherence scanning interferometer (VS-1000, Hitachi Co.). Objective lenses of 50× and 20× were used for cross-sectional profiles and a surface roughness evaluation, respectively. The distance between the sample and the objective lens was scanned vertically with the range of $\pm 10 \mathrm{~mm}$ from the standard focal point. The vertical scan was conducted three times to obtain the averaged microscopic image. The acquired data was analyzed by a software package (VS-viewer, Hitachi Co.). Figure S7 shows the cross-sectional profiles of $\mathrm{SiO}_{2}$ microdisks. The cross-sectional profiles found that the microdisks were slightly tapered. From the slope of the edge of the microdisks, the taper angles were estimated to be $60^{\circ} \pm 10^{\circ}, 60^{\circ} \pm 11^{\circ}, 61^{\circ} \pm$ $12^{\circ}$, and $60^{\circ} \pm 10^{\circ}$ for $L=4,8,11,16 \mu \mathrm{m}$, respectively. Note that the resultant taper angles are limited to the resolution of the magnification of the coherence scanning interferometer. The tapered structure implies that the WGM can leak from the edges of the microdisks through a layer of BSB-Cz deposited on the tapered $\mathrm{SiO}_{2}$ microdisk edges. 

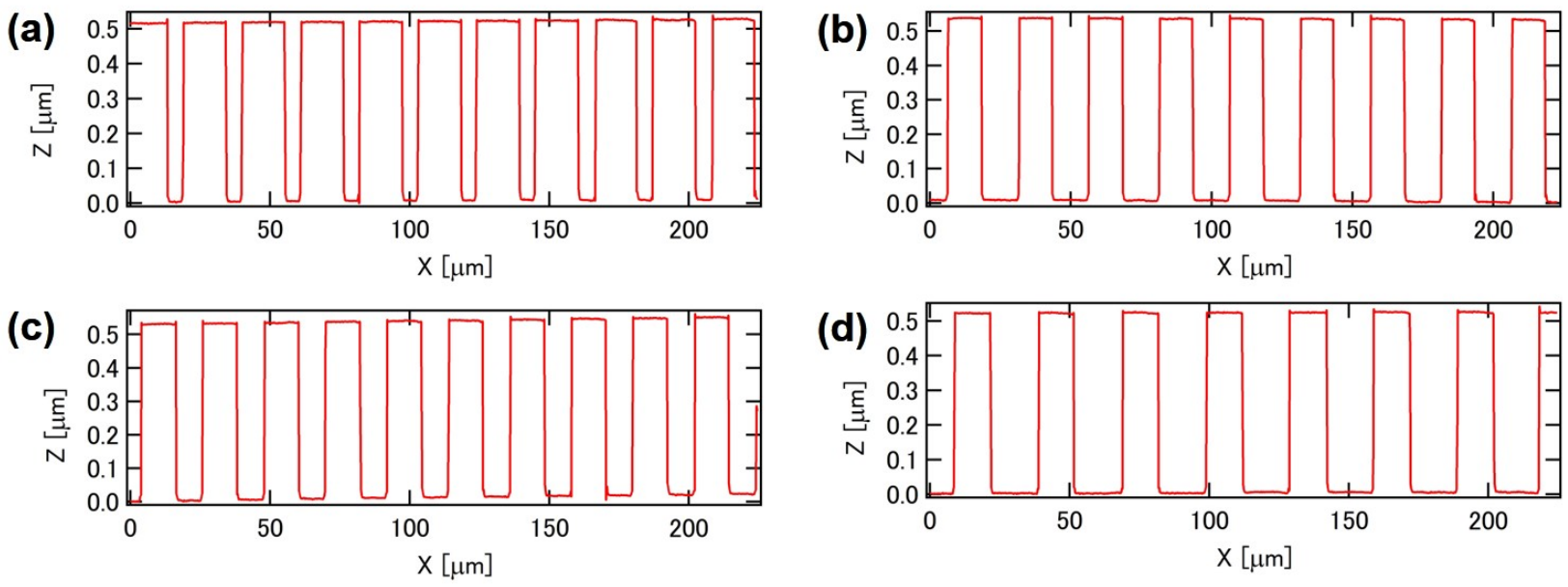

Figure S7. Cross-sectional profiles of (a) $L=4 \mu \mathrm{m}$, (b) $L=8 \mu \mathrm{m}$, (c) $L=11 \mu \mathrm{m}$, and (d) $L=16 \mu \mathrm{m}$.

The coherence scanning interferometer observation revealed that the surface roughness values were less than $1.1 \mathrm{~nm}$ and $0.6 \mathrm{~nm}$ for the top surfaces of $\mathrm{SiO}_{2}$ and $\mathrm{BSB}-\mathrm{Cz}$ microdisks, respectively. However, the roughness of the outer edge of the microdisks are impossible to estimate, meaning that the roughness of the outer edge is possible to be higher than the top surface of the microdisks to assist the leakage of WGMs from the resonators.

Angular dependent PL measurements. Figure S8 shows the dependence of the PL spectra on a detector angle. The experimental setup is shown in Fig. S8a. The excitation light and the monochromator with the CCD photo detector were the same as the microspectroscope system described in the main text. The samples were mounted on a half cylinder prism via a matching oil. A detector angle is an angle between normal to a substrate and a direction of PL detection. Collimator lens, which is connected to the monochromator through a fiber cable, collected PL emission from the samples. A polarizer and a long-pass filter with a cut-off wavelength of $400 \mathrm{~nm}$ were inserted between a sample space and the collimator. The polarization direction is shown in Fig. S8a. The measurements were conducted with a step of $10^{\circ}$. In the samples of $L=8 \mu \mathrm{m}$, PL intensity increased at $30^{\circ}$. Because the Brewster's angle at the organic/ $\mathrm{SiO}_{2}$ interface is $26^{\circ}$, the light intensity enhancement is attributed to a leaky mode at the Brewster's angle. ASE with a WGM resonance was observed even at the Brewster's angle in the sample of $L=8 \mu \mathrm{m}$ under a condition that exhibits WGM peaks. This result 
implies that light amplification with a WGM resonance occurs even at much wider than a propagation angle for optical waveguide mode.
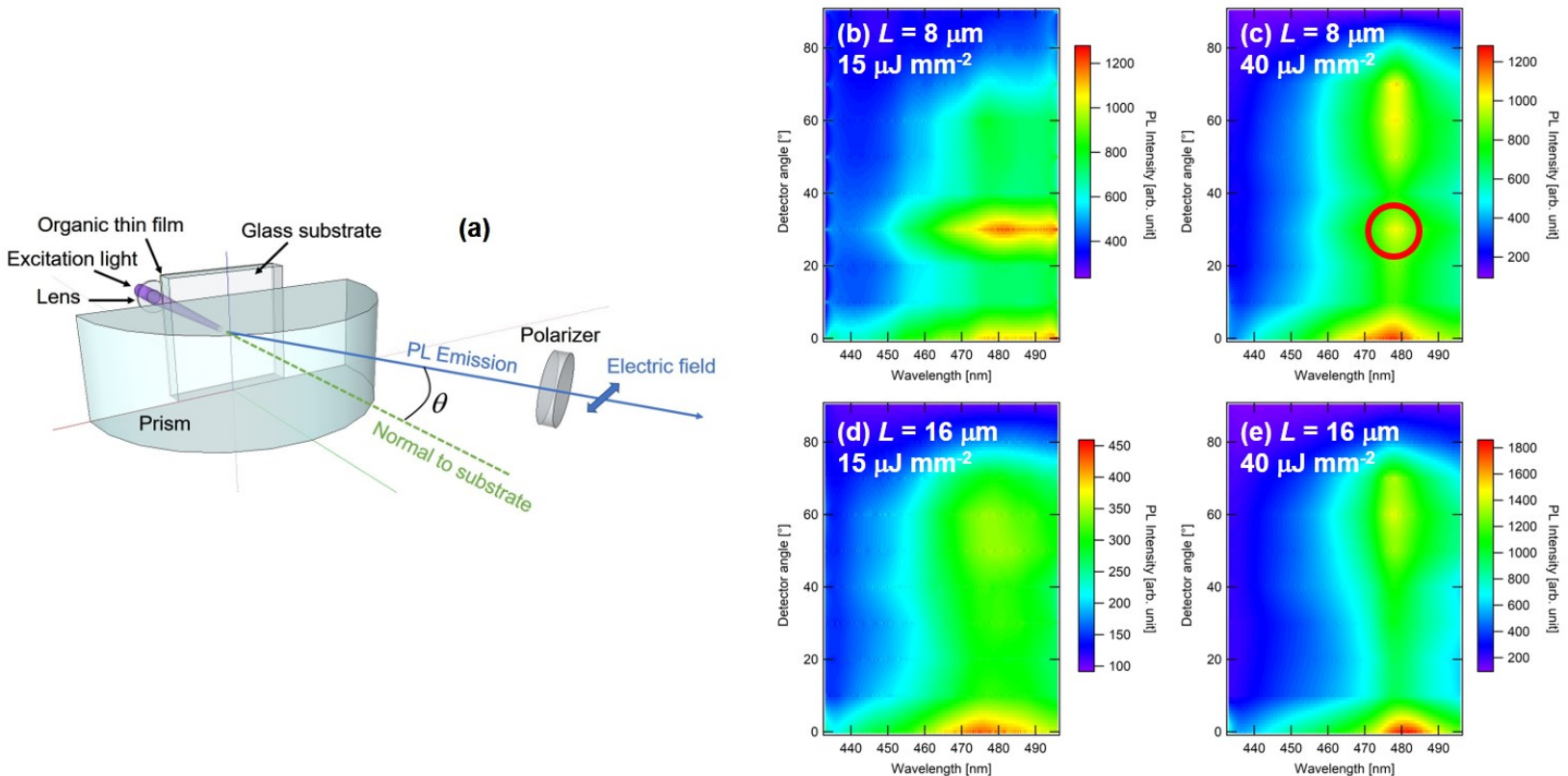

Figure S8. (a) Experimental setup for angular dependence PL measurements. The images of (b) and (c) were the results in $L=8 \mu \mathrm{m}$, while (d) and (e) were the results in $L=16 \mu \mathrm{m}$. The excitation intensities were either $15 \mu \mathrm{J} \mathrm{mm}^{-2}$ [(b) and (d)] or $40 \mu \mathrm{J} \mathrm{mm}^{-2}$ [(c) and (e)]. The circle in (c) indicates a WGM resonance at the Brewster's angle.

Estimation of $\mathbf{Q}$ factors. Figure S9 shows the fitting results of the PL spectra. We fitted the WGM peaks by convolution with a Lorentzian oscillator. We consider that a background of the WGM peaks is well fitted by Tanc-Lorentz functions and Gaussian functions, but 4-6 Lorentzian reproduced the experiments; herein, we do not focus on each parameter of functions for the background. In Figs S6c and S6d, several small peaks of fine structures could not be analyzed, while relatively large peaks were successfully fitted by Lorentzian. We calculated Q factors by $\omega_{0} / \mathrm{FWHM}$, where $\omega_{0}$ is a peak angular frequency of each WGM peak. The calculated Q factors for $L=16,11,8,4$ were $378 \pm 63,457 \pm 197$, $871 \pm 359,891 \pm 368$, respectively. 

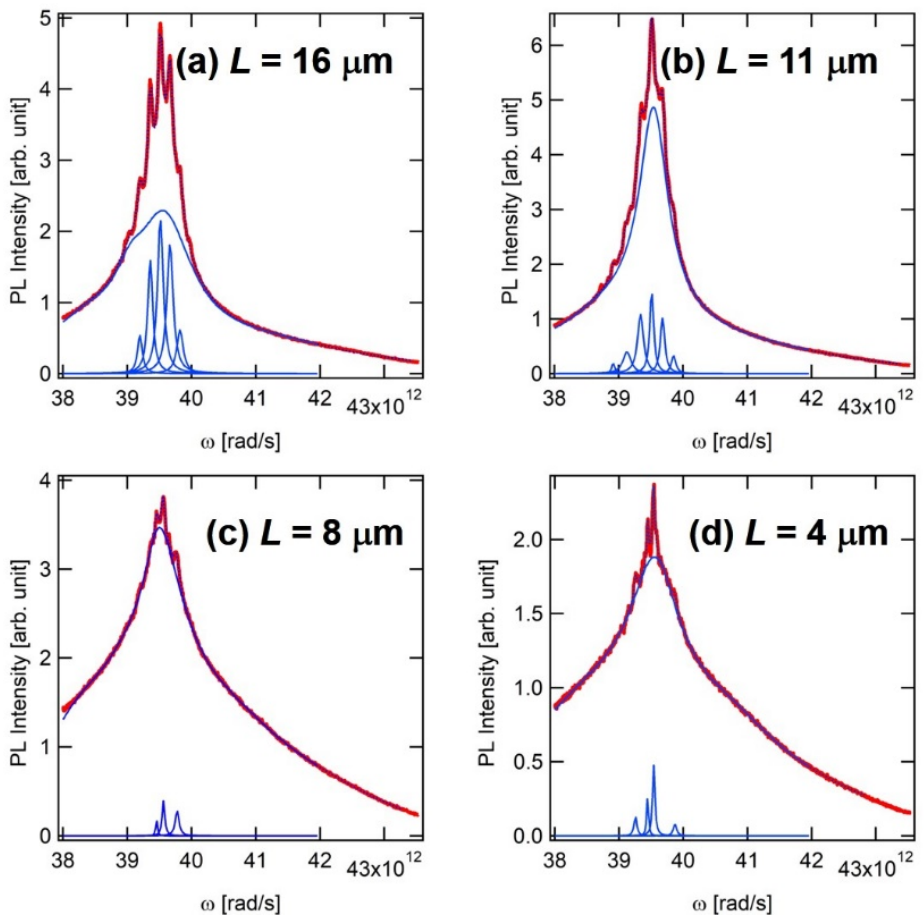

Figure S9. Fittings of PL spectra in (a) $L=16 \mu \mathrm{m}$, (b) $L=11 \mu \mathrm{m}$, (c) $L=8 \mu \mathrm{m}$, and (d) $L=4 \mu \mathrm{m}$. The spectra are the same as the spectra in Fig. 2, but the horizontal axis is angular frequency. The excitation intensity is either $150 \mu \mathrm{J} \mathrm{mm}^{-2}$ [(a), (b), and (d)] or $121 \mu \mathrm{J} \mathrm{mm}^{-2}$ (d). Thin Solid lines and thin broken lines are the fitting results and the deconvoluted spectra, respectively. 